\title{
Facilitadores para a prática de atividade física no lazer em adolescentes
}

\section{Facilitators for physical activity at leisure in adolescents}

\section{AUTORES \\ Edina Maria de Camargo $0^{1,2,3}$ (D) \\ Hermes Koller de Paiva ${ }^{1,3}$ \\ Henrique Lustosa de Mello Pacheco ${ }^{4}$ (D) \\ Wagner de Campos ${ }^{1,2}$ (D) \\ 1 Universidade Federal do Paraná (UFPR), Setor de Ciências Biológicas, Programa de Pós-Graduação em Educação Física, Curitiba, Paraná, Brasil. 2 Centro de estudos em atividade física e saúde (CEAFS), Curitiba, Paraná, Brasil. \\ 3 Grupo de pesquisa em atividade física e qualidade de vida (GPAQ), Curitiba, Paraná, Brasil \\ 4 Pontifícia Universidade Católica do Paraná, Curso de Educação Física, Curitiba, Paraná, Brasil.}

\section{CONTATO}

Edina Maria de Camargo

edinacamargo@gmail.com

Rua Coração de Maria, 92, Campus Jardim

Botânico, Curitiba, Paraná, Brasil.

CEP: 80210-132.

DOI

10.12820/rbafs.v.22n6p561-67

\section{(ब) $\Theta \Theta \Theta$}

Copyright: This is an open-access article distributed under the terms of the Creative Commons Attribution License ${ }^{\circledR}$, which permits unrestricted use, distribution, and reproduction in any medium, provided that the original author and source are credited.

\begin{abstract}
RESUMO
O objetivo do estudo foi identificar os facilitadores para a prática de atividade física no lazer em adolescentes do ensino médio de São José dos Pinhais (PR). Foi realizada a técnica de grupos focais, da pesquisa qualitativa, seguindo um roteiro semiestruturado, com adolescentes de 14 a 17 anos de idade ( $50 \%$ do sexo feminino, média de idade de $15,5 \pm 1,5$ ) de escolas públicas e privadas. Ao total foram realizados 12 grupos focais, sendo metade para cada sexo e tendo adolescentes ativos e inativos fisicamente. Para a análise dos dados foi utilizado a análise de conteúdo e a estatística descritiva para contabilizar os relatos. Os resultados foram analisados separadamente por tipo de escola e sexo. Os facilitadores mais relatados foram: apoio social dos amigos (12,2\%), apoio social dos pais $(11,7 \%)$, ter habilidades $(11,1 \%)$, educação física trabalhar as habilidades motoras e físicas $(10,3 \%)$ e praticar atividade física no contraturno das aulas (7,6\%). Para maximizar os níveis de atividade física no lazer em adolescentes, deve-se levar em consideração os facilitadores encontrados nesse estudo, que dizem respeito ao apoio social e a prática efetivamente, seja ela para o aprendizado de habilidades motoras e físicas, ou para o aprimoramento, tanto nas aulas de educação física escolar quanto nas atividades físicas no contraturno.
\end{abstract}

Palavras-chave: Atividade motora; Exercício; Adolescente; Grupos focais.

\begin{abstract}
The objective of the study was to identify the facilitators for the practice of leisure physical activity in high school adolescents from São José dos Pinhais (PR). A focus group technique was used for qualitative research, followed by a semi-structured script, with adolescents between 14 and 17 years of age $(50 \%$ females, mean age $15.5 \pm 1.5$ ) from public and private schools. Twelve focus groups were performed, half for each sex and adolescents with different levels of physical activity. For the analysis of the data, we used the content analysis and the descriptive statistics to account for the reports. The results were stratified by type of school, gender and general, with the most reported being: social support from friends (12.2\%), parental social support (11.7\%), having skills (11.1\%), education physical and motor skills (10.3\%) and practice physical activity in the back of class (7.6\%). In order to maximize physical activity levels in adolescent leisure, the facilitators found in this study, which concern social support and practice, should be taken into account. Whether it is for the learning of physical and motor skills or for improvement, both in physical education classes and in physical activities in the back of class.
\end{abstract}

Keywords: Motor activity; Exercise; Adolescent; Focus groups.

\section{Introdução}

Estudos indicam que a prática regular de atividade física entre adolescentes está associada a um menor risco no excesso de peso corporal, melhora na capacidade musculoesquelética, indicadores metabólicos, saúde óssea e capacidade pulmonar ${ }^{6}$. Adolescentes fisicamente ativos possuem maior chance de permanecerem ativos na idade adulta, conferindo os benefícios a longo prazo ${ }^{7-9}$.

As evoluções tecnológicas e sociais promoveram grandes consequências nos hábitos e comportamentos das pessoas $^{1,2}$, em especial nos adolescentes, por se tratar de uma geração com maior contato com essa realidade tecnológica (videogames e computadores) ${ }^{3,4}$. Entre os efeitos observados, o aumento da inatividade física nesta faixa etária tem preocupado pesquisadores e especialistas ${ }^{1,4,5}$.

No Brasil, os dados disponíveis sobre adolescentes têm demostrado elevadas prevalências de inatividade física $^{1,10}$. Estimativas nacionais do período de 20132014 indicaram que metade dos adolescentes não prática atividade física no lazer, nos níveis recomendados ${ }^{1}$. 
As regiões Sul e Nordeste estão entre as que possuem as maiores prevalências de adolescentes que não cumprem a recomendações de atividade física $(56,1 \%$ e $57 \%$, respectivamente) e $25 \%$ não realizam atividade física ${ }^{1,10}$.

As atuais evidências permitem demonstrar informações importantes sobre o nível de atividade física e os fatores associados para a prática de atividade física ${ }^{8,9}$, tais como: sexo, idade, classe econômica, apoio social e a percepção de barreiras ${ }^{11-13}$. No entanto, poucos estudos têm se preocupado em descrever quais são os facilitadores para a prática de atividades físicas. Facilitadores são fatores que poderiam oportunizar ou viabilizar a prática de atividade física. Uma vez descritos na literatura, essas informações podem melhorar a compreensão sobre o tema e direcionar intervenções específicas para maximizar a prática de atividade física em adolescentes.

Diante da elevada prevalência de inatividade física entre adolescentes e os agravos associados a comportamento, a identificação dos facilitadores é fundamental para que as intervenções elaboradas para aumentar os níveis de atividade física em adolescentes possam ser mais efetivas ${ }^{14,15}$. Sendo assim, o objetivo do presente estudo foi identificar os facilitadores para a prática de atividade física no lazer em adolescentes do ensino médio de São José dos Pinhais (PR).

\section{Métodos}

\section{Características do estudo}

Este estudo caracteriza-se como uma pesquisa descritiva exploratória, com delineamento transversal, utilizando-se da técnica de grupos focais. A técnica de grupos focais foi utilizada para auxiliar a compreensão dos facilitadores para a prática de atividade física em adolescentes. Esta técnica foi utilizada anteriormente em outros estudos, com adolescentes ${ }^{16}$, adultos ${ }^{17}$ e ido$\operatorname{sos}^{18}$ para investigar a prática de atividade física.

\section{Participantes e seleção}

Fizeram parte do estudo escolares do ensino médio de 14 a 17 anos de idade, de ambos os sexos, da rede pública e privada do município de São José dos Pinhais (PR). Essa faixa etária foi definida por que a literatura aponta que na adolescência os níveis de atividade física reduzem. Foram selecionadas de forma intencional seis escolas, sendo três públicas e três privadas. Em cada escola selecionada, os escolares dentro da faixa etária pré-estabelecida (14 a 17 anos de idade) foram convidados a responder um questionário sobre a prática de atividade física e informações demográficas. Esse questionário teve o objetivo de pré-selecionar, os alunos ati- vos e não ativos para participar da entrevista com grupos focais. Após a seleção pelo nível de atividade física, aconteceu a seleção intencional dos participantes, pois foram priorizados os alunos que possuíam facilidade de se comunicar, opinar, criticar, tendo em vista que a técnica de grupo focal necessita de participantes comunicativos ${ }^{19}$. Ao total foram realizados 12 grupos focais, sendo metade para cada sexo e tendo adolescentes ativos e inativos fisicamente.

O estudo foi aprovado pelo comitê de ética em pesquisa da Pontifícia Universidade Católica do Paraná - PUCPR (Processo n.o 020622/2014) e todos os participantes aceitaram participar de maneira voluntária. Foi enviado aos pais ou responsáveis dos escolares o Termo de Consentimento Livre e Esclarecido (TCLE), para autorizarem a participação dos mesmos na pesquisa. As entrevistas foram conduzidas entre os meses de agosto, setembro e outubro de 2014.

\section{Elaboração dos grupos focais}

O número de participantes em cada grupo foi determinado de acordo com as recomendações de Stewart e Shamdasani ${ }^{19}$, que sugere que os grupos focais sejam homogêneos, constituídos por, no máximo, 12 integrantes e selecionados de maneira intencional. Um número elevado de participantes dificulta o gerenciamento das entrevistas pelo moderador. Foram tomados os devidos cuidados com relação a aspectos éticos, local da aplicação, experiência do moderador, roteiro e duração da entrevista, bem como o registro das informações ${ }^{19}$.

A moderação dos grupos foi realizada por um único pesquisador, previamente treinado para tal finalidade. As entrevistas seguiram um roteiro semi-estruturado (apresentado no próximo tópico), que teve como base estudos prévios da literatura, com questões abertas e tópicos vinculados aos facilitadores para a prática de atividade física, testado previamente em um estudo piloto. $\mathrm{O}$ piloto foi realizado para testar as informações do roteiro semi-estruturado e avaliar a compreensão por adolescentes. Os adolescentes do estudo piloto compreenderam bem as questões, com isso, o roteiro não teve alterações e foi aplicado nas sessões. Tanto o piloto quanto as entrevistas tiveram duração média de 60 minutos.

As discussões das sessões dos grupos focais foram registradas e gravadas tendo-se ao final de cada entrevista um registro manuscrito das informações importantes e um registro em áudio na íntegra. A gravação foi iniciada mediante autorização dos participantes e finalizada após o encerramento da discussão. Posteriormente, os arquivos foram transcritos na íntegra, atri- 
buindo-se a cada participante um código (por exemplo, P1, P2, P3 ...). Após a transcrição dos relatos na íntegra, as frases (transcritas) foram agrupadas de acordo com os seus temas e foi criado códigos para cada grupo, esses códigos seriam os facilitadores (Ex.1. “...se tivesse atividades físicas no contraturno facilitaria, por que na escola minha mãe deixa..." - código= atividade física no contraturno. Ex.2. “...eu não consigo jogar, eu queria, mas não tenho habilidade, nunca tive coordenação...”código $=$ habilidade motora/física).

\section{Instrumento - Prática de atividade física em adolescentes}

A prática de atividades físicas realizadas pelos adolescentes foi avaliada utilizando o Questionário de Atividade Física para Adolescentes (QAFA) ${ }^{20}$. Este questionário foi primeiramente desenvolvido no formato de checklist por Sallis et $\mathrm{al}^{21}$. para adolescentes norte americanos, traduzido e adaptado para a adolescentes brasileiros por Farias Júnior ${ }^{20}$. O questionário é composto por uma lista de 24 atividades físicas, de intensidade moderada a vigorosa, em que o adolescente relata qual a frequência e a duração que realizou cada atividade. Existe a possibilidade do adolescente adicionar atividades além das listadas. Em adolescentes brasileiros o questionário apresentou boa reprodutibilidade $(\mathrm{CCI}=$ 0,88; IC95\%: 0,84-0,91) e validade concorrente quando comparado com recordatório de 24 horas ( $r=0,62$; $\mathrm{p}<0,001)^{20}$. Esse questionário teve o objetivo de pré-selecionar, os alunos ativos e não ativos para participar da entrevista com grupos focais. Foram considerados ativos os adolescentes que cumpriram a recomendação de $420 \mathrm{~min} / \mathrm{semana}$ de atividade física.

\section{Instrumento - Roteiro Semi-estruturado}

O roteiro foi dividido em duas partes: a primeira parte foi a apresentação do mediador ao grupo e objetivos do estudo. A segunda parte caracterizou-se pelo estímulo à discussão, e para isso foi utilizado imagens de adolescentes que praticavam atividade física em diversas situações, onde os adolescentes deveriam relatar os possíveis facilitadores que oportunizam, estimulam a prática de atividade física.

\section{Informações demográficas}

Foi verificado o sexo (masculino e feminino), a idade (categorizado em 14 e 15 anos; 16 e 17 anos de idade) e a classe econômica, com base na posse de bens na residência, escolaridade do responsável financeiro pelo domicílio e presença de empregados domésticos $(\mathrm{ABEP})^{22}$.
Para análise, as classes econômicas foram categorizadas em "alta" (classes $\mathrm{A}+\mathrm{B})$ e "baixa" (classes $\mathrm{C}+\mathrm{D}+\mathrm{E})$.

\section{Análise dos dados}

As informações obtidas nos grupos focais foram analisadas por meio de uma abordagem qualitativa com o emprego da análise de conteúdo ${ }^{19}$. Esse procedimento consiste na leitura extensiva das transcrições buscando encontrar temas para a realização do agrupamento. Esses temas são agrupados com códigos, de acordo com a literatura, que são os facilitadores. Dessa maneira, a leitura e releitura das transcrições permitiu a identificação dos possíveis facilitadores. Após a análise do conteúdo foi realizado a estatística descritiva para obtenção das frequências relativa e absoluta dos códigos, analisados separadamente por sexo e por tipo de escola.

\section{Resultados}

Participaram da amostra 144 adolescentes (50,0\% do sexo feminino), metade da amostra relatou idade entre 14 e 15 anos, cumprir a recomendação de atividade física (420 min/semana), pertencer a escola pública e em relação à classe econômica, $64,0 \%$ relatou pertencer ao nível alto (classes A e B). Não houve perdas e/ou recusas.

Ao todo foram identificados 1.062 relatos durante os 12 grupos focais realizados nas escolas. Os relatos foram contabilizados e analisados separadamente por tipo de escola e sexo (Tabela 1 e 2) e de maneira geral (Tabela 3), sendo os mais relatados: apoio social dos amigos (12,2\%), apoio social dos pais $(11,7 \%)$, habilidade motora/física (11,1\%), educação física trabalhar habilidades motoras e físicas (10,3\%) e praticar atividade física no contraturno das aulas (7,6\%). Em relação ao tipo de escola, pública e privada: apoio social de amigos $(10,8 \%$ versus $14,9 \%)$, apoio social de pais $(10,2 \%$ versus $14,4 \%)$, ter habilidade motora/física $(9,6 \%$ versus $13,9 \%)$, educação física trabalhar habilidades $(8,9 \%$ versus $12,8 \%)$ foram as mais mencionadas. Em relação ao sexo, os facilitadores coincidiram com os contabilizados na classificação geral. No Quadro 1, há exemplos de relatos, referente aos facilitadores mais citados

\section{Discussão}

Os facilitadores para a prática de atividade física no lazer em adolescentes, dizem respeito, tanto nas escolas privadas quanto nas públicas, a ter apoio social de pais e amigos, ter habilidades para realizar atividades físicas e a importância de desenvolver essas habilidades seja na educação física escolar, com profissional ou com amigos. Ainda, atividades oferecidas no contraturno 
Quadro 1 - Seleção de alguns dos relatos citados pelos adolescentes nas sessões de grupos focais, São José dos Pinhais (PR), 2014.

Apoio social de amigos: "... se meus amigos vão comigo é muito melhor. Eu prefiro, quando eles vão comigo, eles me animam, me incentivam ..." (relato de um adolescente de escola pública) ou ".... se a gente tem um grupo é muito melhor, fazer sozinho é chato, mas quando os amigos estão juntos, até o tempo passa mais rápido, ficamos horas..." (relato de uma adolescente de escola privada).

Apoio social de pais: "... eu faço por que meus pais pagam e me levam todo dia, mas eu sei que muitos dos meus amigos não tem essa oportunidade, esse incentivo dos pais. Pra mim, isso ajuda." (relato de uma adolescente de escola privada) ou "...na verdade, meus pais fazem atividades físicas, eles gostam, sempre incentivaram a gente a fazer, desde ir junto, participar de competiçôes, eles gostam de assistir minhas lutas...” (relato de um adolescente de escola pública).

Ter habilidade (motora/física): “...se eu tivesse aprendido, com certeza eu jogaria, mas eu não sei...” (relato de uma adolescente de escola particular) ou “...eu queria, mas não sei, não tenho jeito. Não consigo jogar igual meus amigos, não tenho coordenação... mas eu queria saber, claro que eu queria...” (relato de um adolescente de escola pública) ou "... Eu já sonhei uma vez que eu jogava muito bem, que era o melhor da sala. Mas na verdade não sou. Queria aprender...queria saber jogar bem..." (relato de um adolescente de escola privada).

Educação física trabalhar habilidades (motoras/físicas): “...não dá tempo de aprender na aula de educação física...”"(relato de um adolescente de escola pública)ou “...eu tenho vergonha de fazer na aula, por que eu não sei e não dá tempo de aprender..." (relato de uma adolescente de escola privada) ou "..o professor não consegue ensinar a gente, o tempo é curto. E eu tenho vergonha de fazer, por que não sei. Então, não jogo por que não sei. E na aula não dá tempo de ensinar e aperfeiçoar. Então já desisti de aprender..." (relato de um adolescente de escola pública).

Praticar atividade física no contraturno das aulas: . "...Tinha que ter atividades para quem não sabe, na escolinha de esporte todo mundo sabe. O professor só seleciona quem sabe para participar..." (relato de um adolescente de escola pública)ou "... Tinha que ter mais atividades no contraturno, atividades de meninas, só tem esporte..." (relato de uma adolescente de escola pública) ou "...Eu sei que é bastante aluno para aprender na aula de educação física, mas se tivesse um outro horário para aprender, numa dessa, eu seria mais ativo. Por que na verdade não gosto de estar fora do peso..." (relato de um adolescente de escola privada).

Tabela 1 - Facilitadores para a prática de atividade física no lazer em adolescentes de escola pública de São José dos Pinhais - Paraná (72 adolescentes -687 relatos).

\begin{tabular}{|c|c|c|c|c|c|c|}
\hline \multirow{3}{*}{ Facilitadores } & \multicolumn{6}{|c|}{ Escolas públicas } \\
\hline & \multicolumn{2}{|c|}{ Masculino $(\mathrm{n}=36)$} & \multicolumn{2}{|c|}{ Feminino $(n=36)$} & \multicolumn{2}{|c|}{ Total $(n=72)$} \\
\hline & $\mathrm{n}$ & $\%$ & $\mathrm{n}$ & $\%$ & $\mathrm{n}$ & $\%$ \\
\hline Apoio social dos amigos & 30 & 8,8 & 44 & 12,6 & 74 & 10,8 \\
\hline Apoio social dos pais & 29 & 8,6 & 41 & 11,8 & 70 & 10,2 \\
\hline Ter habilidade motora e física & 36 & 10,6 & 30 & 8,6 & 66 & 9,6 \\
\hline Aula de educação física trabalhar habilidades motoras e físicas & 31 & 9,1 & 30 & 8,6 & 61 & 8,9 \\
\hline Praticar atividade física no contraturno das aulas & 23 & 6,8 & 36 & 10,3 & 59 & 8,6 \\
\hline Alguém que ensine a fazer atividade física & 28 & 8,3 & 30 & 8,6 & 58 & 8,4 \\
\hline Atividades físicas específicas (em relação ao sexo, faixa etária) & 12 & 3,5 & 14 & 4,0 & 26 & 3,8 \\
\hline Possuir espaços públicos de lazer na vizinhança & 30 & 8,8 & 19 & 5,5 & 49 & 7,1 \\
\hline Local seguro & 6 & 1,8 & 34 & 9,8 & 40 & 5,8 \\
\hline Gostar da atividade física que faz & 26 & 7,7 & 11 & 3,2 & 37 & 5,4 \\
\hline Apoio dos professores de educação física & 17 & 5,0 & 9 & 2,6 & 26 & 3,8 \\
\hline Se não houvesse bullying & 12 & 3,5 & 14 & 4,0 & 26 & 3,8 \\
\hline Possuir equipamentos em casa & 9 & 2,7 & 11 & 3,2 & 20 & 2,9 \\
\hline Aplicativos que incentivam/Rede social que cria eventos & 12 & 3,5 & 7 & 2,0 & 19 & 2,8 \\
\hline Ter material esportivo nas escolas/Liberação dos materiais esportivos & 14 & 4,1 & 4 & 1,1 & 18 & 2,6 \\
\hline Possuir atividade física perto de casa (distância) & 12 & 3,5 & 4 & 1,1 & 16 & 2,3 \\
\hline Receber apoio da prefeitura/governo & 8 & 2,4 & 4 & 1,1 & 12 & 1,7 \\
\hline Ter tempo livre & 4 & 1,2 & 6 & 1,7 & 10 & 1,5 \\
\hline Total de relatos (facilitadores) & 339 & 100,0 & 348 & 100,0 & 687 & 100,0 \\
\hline
\end{tabular}

das aulas e, alguém que ensine a fazer, parecem favorecer a prática de atividade física em adolescentes.

A relevância destas informações consiste em contribuir para a reformulação das aulas de educação física, assim como, nas atividades extracurriculares e demais ações relativas à promoção da atividade física em adolescentes. Os profissionais da educação física devem estar atentos ao desenvolvimento de habilidades, visando uma parcela, das aulas de educação física escolar ou das atividades do contraturno, para essa finalidade.

Os dados foram analisados separadamente em escolas públicas e privadas, pois acreditava-se que uma vez que as realidades das escolas são diferentes em relação a estrutura, profissionais, nível socioeconômico do bairro em que a escola esta inserida, os relatos poderiam ser diferentes. Mas, quando analisado separadamente por tipo de escola e sexo, foi possível observar que os resultados foram semelhantes.

$\mathrm{O}$ apoio social dos amigos e pais foram os facilitadores 
Tabela 2 - Facilitadores para a prática de atividade física no lazer em adolescentes de escola privada de São José dos Pinhais - Paraná (72 adolescentes -375 relatos).

\begin{tabular}{|c|c|c|c|c|c|c|}
\hline \multirow{3}{*}{ Facilitadores } & \multicolumn{6}{|c|}{ Escolas privadas } \\
\hline & \multicolumn{2}{|c|}{ Masculino $(n=36)$} & \multicolumn{2}{|c|}{ Feminino $(n=36)$} & \multicolumn{2}{|c|}{ Total $(n=72)$} \\
\hline & $\mathrm{n}$ & $\%$ & $\mathrm{n}$ & $\%$ & $\mathrm{n}$ & $\%$ \\
\hline Apoio social dos amigos & 22 & 13,8 & 34 & 16,6 & 56 & 14,9 \\
\hline Apoio social dos pais & 28 & 17,5 & 26 & 12,7 & 54 & 14,4 \\
\hline Ter habilidade motora e física & 28 & 17,5 & 24 & 11,7 & 52 & 13,9 \\
\hline Aula de educação física trabalhar habilidades motoras e físicas & 22 & 13,8 & 26 & 12,7 & 48 & 12,8 \\
\hline Praticar atividade física no contraturno das aulas & 12 & 7,5 & 10 & 4,9 & 22 & 5,9 \\
\hline Alguém que ensine a fazer atividade física & 9 & 5,6 & 12 & 5,9 & 21 & 5,6 \\
\hline Atividades físicas específicas (em relação ao sexo, faixa etária) & 8 & 5,0 & 10 & 4,9 & 48 & 12,8 \\
\hline Possuir espaços públicos de lazer na vizinhança & 4 & 2,5 & 4 & 2,0 & 8 & 2,1 \\
\hline Local seguro & 4 & 2,5 & 4 & 2,0 & 8 & 2,1 \\
\hline Gostar da atividade física que faz & 0 & 0,0 & 8 & 3,9 & 8 & 2,1 \\
\hline Apoio dos professores de educação física & 6 & 3,8 & 9 & 4,4 & 15 & 4,0 \\
\hline Se não houvesse bullying & 0 & 0,0 & 11 & 5,4 & 11 & 2,9 \\
\hline Possuir equipamentos em casa & 2 & 1,3 & 2 & 1,0 & 4 & 1,1 \\
\hline Aplicativos que incentivam/Rede social que cria eventos & 8 & 5,0 & 16 & 7,8 & 4 & 1,1 \\
\hline Ter material esportivo nas escolas/Liberação dos materiais esportivos & 0 & 0,0 & 4 & 2,0 & 4 & 1,1 \\
\hline Possuir atividade física perto de casa (distância) & 4 & 2,5 & 2 & 1,0 & 6 & 1,6 \\
\hline Receber apoio da prefeitura/governo & 2 & 1,3 & 2 & 1,0 & 4 & 1,1 \\
\hline Ter tempo livre & 1 & 0,6 & 1 & 0,5 & 2 & 0,0 \\
\hline Total de relatos (facilitadores) & 160 & 100,0 & 205 & 100,0 & 375 & 100,0 \\
\hline
\end{tabular}

Tabela 3 - Facilitadores para a prática de atividade física no lazer em adolescentes de escola pública e privada de São José dos Pinhais - Paraná (144 adolescentes - 1062 relatos).

\begin{tabular}{lcc}
\hline & \multicolumn{2}{c}{$\begin{array}{c}\text { Todos } \\
\text { Facilitadores }\end{array}$} \\
& $\begin{array}{c}(\mathrm{n}=144 \\
\text { adolescentes})\end{array}$ \\
\cline { 2 - 3 } & $\mathrm{n}$ & $\%$ \\
\hline Apoio social dos amigos & 130 & 12,2 \\
Apoio social dos pais & 124 & 11,7 \\
Ter habilidade motora e física & 118 & 11,1 \\
Aula de educação física trabalhar habilidades motoras e físicas & 109 & 10,3 \\
Praticar atividade física no contraturno das aulas & 81 & 7,6 \\
Alguém que ensine a fazer atividade física & 79 & 7,4 \\
Atividades físicas específicas (em relação ao sexo, faixa etária) & 74 & 7,0 \\
Possuir espaços públicos de lazer na vizinhança & 57 & 5,4 \\
Local seguro & 48 & 4,5 \\
Gostar da atividade física que faz & 45 & 4,2 \\
Apoio dos professores de educação física & 41 & 3,9 \\
Se não houvesse bullying & 37 & 3,5 \\
Possuir equipamentos em casa & 24 & 2,3 \\
Aplicativos que incentivam/Rede social que cria eventos & 23 & 2,2 \\
Ter material esportivo nas escolas/Liberação dos materiais esportivos & 22 & 2,1 \\
Possuir atividade física perto de casa (distância) & 22 & 2,1 \\
Receber apoio da prefeitura/governo & 16 & 1,5 \\
Ter tempo livre & 12 & 1,1 \\
Total de relatos (facilitadores) & 1062 & 100,0 \\
\hline
\end{tabular}

mais citados pelos adolescentes da escola pública e privada. Prado et $\mathrm{al}^{25}$ encontrou, em seu estudo com ado- lescentes da rede pública de Curitiba, que possuir amigos que convidam, acompanham, incentivam, acham bom e gostam de praticar atividade física aumentam a probabilidade do adolescente atingir as recomendações de atividade física, independentemente do sexo ${ }^{25}$. Quando verificado o apoio da família (pais, irmãos), um estudo de revisão sistemática ${ }^{26}$ mostrou que existe uma grande influência do pai e da mãe com suas filhas, tanto para inatividade quanto para serem ativas fisicamente ${ }^{26}$. O apoio social tem demonstrado ser capaz de aumentar os níveis de atividade física em adolescentes ${ }^{13}$ e jovens adultos $^{27}$, pois esse mecanismo de apoio sugere estímulo, praticar junto, transportar para a atividade, assistir e conversar sobre a atividade. Ações práticas podem ser realizadas, partindo do interesse e mobilização dos próprios pais em relação a prática, por meio do convite e/ou incentivo e/ou praticando junto ${ }^{13}$.

Ter habilidade motoralfísica foi outro facilitador que apareceu com grande frequência para os adolescentes de São José dos Pinhais (PR). As habilidades de crianças e adolescentes são foco de estudos dos pesquisadores há décadas ${ }^{23}$. $\mathrm{E}$ os estudos têm mostrado que o estímulo (incentivar a prática de atividade física) é um dos principais influenciadores na aquisição e performance dos padrões motores fundamentais e físicos ${ }^{23}$. Com base nisso, a aquisição e performance dos padrões motores fundamentais devem ser estimulados e treinados $\mathrm{du}^{-}$ 
rante a infância e adolescência. Quando comparado as habilidades motoras fundamentais de adolescentes em relação ao sexo, estudos tem evidenciado a superioridade de desempenho do sexo masculino em relação ao feminino ${ }^{24}$. Talvez, oportunidades diferenciadas por sexo, para a prática de atividade física, podem estar sendo oferecidas para adolescentes. Sendo assim, em práticas motoras realizadas na educação física escolar, no contraturno, em clubes e demais locais, é essencial incluir todos sem distinção, promovendo a equidade no aprendizado de habilidades motoras. $O$ fato de "não conseguir realizar exercícios físicos/ não saber como faz" foi reportado com uma das principais barreiras para a prática de atividade física em um estudo de prevalência conduzido com adolescentes na cidade de Curitiba ${ }^{12}$. Dessa maneira, trabalhar o desenvolvimento de habilidades físicas e motoras pode facilitar a adesão a prática, uma vez que estaria contribuindo para o aprendizado.

Outro facilitador citado pelos adolescentes diz respeito as aulas de educação física, educação física trabalhar as habilidades motoras e físicas. Os achados do presente estudo revelam que o tempo destinado à disciplina de educação física nas escolas não está sendo adequado para que nossas crianças e adolescentes desenvolvam habilidades suficientes para realizar jogos, brincadeiras, esportes e demais. Consequentemente, o que deveria ser um facilitador para a prática de atividade física acaba se tornando uma barreira pois, se não aprendem corretamente não se sentem competentes para tais atividades, diminuindo assim a autoeficácia para a atividade física. É necessário ter um olhar cuidadoso, sobre o aprendizado da educação física escolar. $\mathrm{O}$ esporte é um dos conteúdos principais das aulas de educação física (futebol, voleibol, basquetebol e handebol) durante todo o ensino fundamental $^{28}$. No entanto, nossas adolescentes reportam dificuldade ao realizar essas atividades. E ainda, relatam que se tivessem aprendido com certeza seriam ativos.

É necessário repensar nas aulas de educação física escolar como uma ferramenta de aprendizagem para o comportamento ativo e ainda, oportunizar o acesso a outras práticas, como por exemplo, esportes com raquetes (badminton, tênis de mesa, tênis de quadra), esportes radicais (skate, slackline), as lutas (capoeira, judô, sumô), as ginásticas (acrobática, de academias). Quanto ao papel da escola na promoção de atividade física, espera-se que ela sirva de elemento propulsor destas iniciativas além das experiências que as aulas de educação física proporcionam. Sugere-se que sejam desenvolvidas atividades físicas no contra turno para ambos os sexos, que aprimorem as habilidades motoras e incentivem a prática corporal (esportes, ginásticas, lutas, dança, jogos). Ainda, que a escola possa ofertar atividades aos finais de semana como jogos recreativos e competitivos e oficinas para a apresentação de novas modalidades, como por exemplo, o aprendizado de futsac, peteca. Da mesma forma, recreios e intervalos podem ser utilizados para desenvolver atividades estruturadas.

Essas estratégias colaboram para os outros facilitadores mais citados: praticar atividade física no contraturno e alguém que ensine a fazer. Em relação as atividades físicas no contraturno, essas atividades são uma excelente oportunidade para aprimorar e/ou melhorar as habilidades de crianças e adolescentes, desenvolver o gosto pela prática da atividade física, junto ao seu grupo etário, com a colaboração da família e a participação dos amigos. E ainda inserir na vida do adolescente a participação em jogos recreativos e/ou competitivos. Portanto, é essencial que as escolas possuam atividades físicas no contraturno das aulas, que atendam meninos e meninas, sem distinção, para promover a equidade no aprendizado de habilidades motoras e físicas de crianças e adolescentes.

A presente investigação apresenta algumas limitações. Para a técnica de grupos focais é utilizado a seleção intencional dos participantes, com base nisso, a seleção dos participantes que não sejam pró-ativos (falem pouco, não discutam, não exponham suas opiniões) pode ser considerado uma limitação, pois o número de relatos depende da discussão, das opiniões expostas. Ainda, deve-se considerar que o estudo foi realizado em único município, o que não permite a extrapolação dos resultados devido à grande diferença de indicadores sociais e ambientais para demais regiões. Como pontos fortes, a utilização dessa técnica permitiu auxiliar a compreensão dos facilitadores para a prática de atividade física em adolescentes.

Os facilitadores para a prática de atividade física no lazer em adolescentes, dizem respeito, tanto nas escolas privadas quanto nas públicas, a ter apoio de pais e amigos, ter habilidades para realizar atividades físicas e a importância de desenvolver essas habilidades seja na educação física escolar, com profissional ou com amigos. Ainda, atividades oferecidas no contraturno das aulas e, alguém que ensine a fazer, também parecem oportunizar a prática da atividade física no lazer.

\section{Conflito de interesses}

Os autores declaram não haver conflito de interesses.

\section{Contribuição dos autores}

Camargo EM participou da concepção inicial do estudo, revisão da 
literatura, coleta de dados, análise dos dados e redação do artigo em todas as suas etapas. Paiva HK participou da revisão da literatura, coleta de dados, análise dos dados e redação do artigo em todas as suas etapas. Pacheco HLM participou da coleta dos dados e revisão da literatura. Campos W foi responsável pela revisão crítica do manuscrito. Todos os autores aprovaram a versão final do manuscrito.

\section{Referências}

1. Cureau FV, Silva TLN, Bloch KV, Fugimori E, Belfort DR, et al. ERICA: Leisure-time physical inactivity in Brazilian adolescents. Rev Saúde Pública. 2016;50(1):1s-11s.

2. Kong IG, Lee H-J, Kim SY, Sim S, Choi HG, et al. Physical activity, study sitting time, leisure sitting time, and sleep time are differently associated with obesity in korean adolescents. Medicine. 2015;94(44):e1965-9

3. KatapallyTR, Muhajarine N. Capturing the interrelationship between objectively measured physical activity and sedentary behaviour in children in the Context of Diverse Environmental Exposures. Int J Environ Res Public Health. 2015;12(9):10995-11011.

4. Muhajarine N, Katapally TR, Fuller D, Stanley KG, Rainham D. Longitudinal active living research to address physical inactivity and sedentary behaviour in children in transition from preadolescence to adolescence. BMC Public Health. 2015;15(1):495-593.

5. NCD Risk Factor Collaboration. Trends in adult body-mass index in 200 countries from 1975 to 2014: a pooled analysis of 1698 population-based measurement studies with $19 \cdot 2$ million participants. Lancet. 2016;387(10026):1377-96.

6. Farias ES, Gonçalves EM, Morcillo AM, Guerra-Junior G, Amancio OMS. Effects of Programmed Physical Activity on Body composition in post-puberal schoolchildren. J Pediatr. 2015;91(2):122-9.

7. Hallal PC, Azevedo MR, Reichert FF, Siqueira FV, Araújo CLP, Victora CG, et al. Who, when, and how much? Epidemiology of walking in a middle-income country. Am J Prev Med. 2005;28(2):156-61.

8. Hallal PC, Bertoldi AD, Gonçalvez H, Victora CG. Prevalência de sedentarismo e fatores associados em adolescentes de 10-12 anos de idade Prevalence of sedentary lifestyle and associated factors in adolescents 10 to 12 years of age. Cad Saúde Pública. 2006;22(6):1277-87.

9. Hallal PC, Knuth AG, Cruz DKA, Mendes MI, Malta DC. Prática de atividade física em adolescentes brasileiros physical activity practice among Brazilian adolescents. Cienc e Saúde Coletiva. 2010;15(2):3035-42.

10. Oliveira JS, Barufaldi LA, De Azevedo Abreu G, Leal VS, Brunken GS, Vasconcelos SML, et al. ERICA: Use of screens and consumption of meals and snacks by Brazilian adolescents. Rev Saúde Pública. 2016;50(1):1s-9s.

11. Farias Junior JC, Reis RS, Hallal PC. Physical activity, psychosocial and perceived environmental factors in adolescents from Northeast Brazil. Cad Saúde Pública. 2014;30(5):941-51.

12. Santos MS, Hino AAF, Reis RS, Rodriguez-Añez CR. Prevalência de barreiras para a prática de atividade física em adolescentes. Rev Bras Epidemiol. 2010;13(1):94-104.

13. Mendonça G, Cheng LA, Mélo EN, Farias Junior JC. Physical activity and social support in adolescents: A systematic review. Health Educ Res. 2014;29(5):822-39.
14. Carlin A, Murphy MH, Gallagher AM. Current influences and approaches to promote future physical activity in 11-13 year olds: a focus group study. BMC Public Health. 2015;15(1270):2601-09.

15. Sallis JF, Cervero RB, Ascher W, Henderson KA, Kraft MK, Kerr J. An Ecological Approach To Creating Active Living Communities. Annu Rev Public Health. 2006;27(1):297-322.

16. Santos MS, Fermino RC, Reis RS, Cassou AC, RodriguezAñez CR. Barreiras para a prática de atividade física em adolescentes. Um estudo por grupos focais Barriers related to physical activity practice in adolescents. Rev Bras Cineantropom e Desempenho Hum. 2010;12(3):137-43.

17. Camargo EM de, Fermino RC, Rodriguez-Añez CR, Reis RS. Barriers and facilitators to bicycle use for transport and leisure among adults. Rev Bras Ativ Fís Saúde. 2014;19(2):256-65.

18. Cassou AC, Fermino RC, Rodriguez-Añez CR, Santos MS, Domingues MR, Reis RS. Barriers to physical activity among brazilian elderly women from different socioeconomic status : A Focus-Group Study. J Phys Act Health. 2011;8(1):126-32.

19. Stewart D and PS. Focus Groups: theory and practice.1990.

20. Farias Júnior JC de, Lopes A da S, Mota J, Santos MP, Ribeiro JC, Hallal PC. Validade e reprodutibilidade de um questionário para medida de atividade física em adolescentes: uma adaptação do Self-Administered Physical Activity Checklist. Rev Bras Epidemiol. 2012;15(1):198-210.

21. Sallis JF, Strilmiller PK, Harsha DW, Feldmam HA, Ehlinger S, Stone EJ, et al. Validation of interviewer-and self- administered physical activity checklists for fifth grade students. J Am Coll Sport Med. 1996;28(7):840-51.

22. ABEP. Associação Brasileira de Empresa de Pesquisa. Critério de classificação economica do Brasil.2013. Available from:<http://www.abep.org/criterio-brasil>[2017 jun12].

23. Machado H, Campos W, Silva SG. Relação entre composição corporal e a performance de padrões motores fundamentais em escolares. Rev Bras Ativ Fís Saúde. 2002;7(1):63-70.

24. Souza MS De, Zanella LW, Bandeira RPF, Silva ACRD, Valentini NC. Meninos e meninas apresentam desempenho semelhante em habilidades motoras fundamentais de locomoção e controle de objeto? Cinergis. 2014;15(4):186-90.

25. Prado CV, Lima AV, Fermino RC, Rodrigues-Añez CR, Reis RS. Apoio social e prática de atividade física em adolescentes da rede pública de ensino: qual a importância da família e dos amigos? Cad Saúde Pública. 2014;30(4):827-38.

26. Piola TS, Martins M, Costa TRA, Campos W. Association of physical activity of adolescents, families and peers: A systematica review. Rev Bras Promoç Saúde. 2015;28(2):281-9.

27. Paiva HK De, Camargo EM de, Silva AA de P, Reis RS. Association between intention for physical activity practice, social support and physical activity. Rev Bras Cineantropom Desempenho Hum. 2016;18(6):667-77.

28. Fortes M de O, Azevedo MR, Kremer MM, Hallal PC. A educação física escolar na cidade de Pelotas-RS: contexto das aulas e conteúdos. Rev da Educ Fis. 2012;23(1):69-78.

29. Sa TH, Garcia LMT, Claro RM. Frequency, distribution and time trends of types of leisure-time physical activity in Brazil, 2006-2012. Int J Public Health. 2014;59(6):975-82.

Recebido: 09/04/2017

Aprovado: 22/12/2017

\section{Como citar este artigo:}

Camargo EM, Paiva HK, Pacheco HLM, Campos W. Facilitadores para a prática de atividade física no lazer em adolescentes. Rev Bras Ati Fis Saúde. 2017;22(6):561-67. DOI: 10.12820/rbafs.v.22n6p561-67. 